MINI LITERATURE REVIEW:

\title{
THE LITERATURE REVIEW OF ELECTRICITY EFFECTS ON CELL PHYSIOLOGY AND RELATED NEW ELECTRICAL TREATMENT MODALITIES
}

\section{Authors:}

\section{Simdi, Ertunc- Uludag University}

\section{E-mail: ertuncsimdi@gmail.com}

\section{Ozcan Kader- Istanbul University}

\section{E-mail: kaderozcan33@gmail.com}

\begin{abstract}
This paper is a mini literature review about electromagnetic field's effects on cells, tissues and new treatment modalities. We have reviewed a papers which have been published in high quality journals in the last 5 years as two authors. This review's aim is to be a resource for experimental studies about electricity's effects on cell biology and pathophysiology. As a result of this literature review, we found that especially extremely low electric frequency and intermediate frequency fields have very important pathophysiological effects. We have mentioned four important expressions on this subject; electroporation, electrofusion, electrochemotherapy, gene electrotransference. Two different new treatment approaches have been developed by use of these two important electrical waves. First is tumor treating fields and the other is pulsed electric fields. Most studies in literature have been done with extremely low frequency and pulsed electric fields. In terms of diseases, most studies are about glioblastoma multiforme and malign melanoma.Most studies in literature have been done with extremely low frequency and pulsed electric fields. In terms of diseases, most studies are about glioblastoma and melanoma.
\end{abstract}

Keywords: Electricity, tumor treating fields, extremely low frequency, pulsed electric fields, new cancer treatment modalities, glioblastoma, electroporation, electrofusion, electrochemotherapy, gene electrotransference

\section{INTRODUCTION}

In this paper, it was our goal to make literature research what kind of effect each frequency of electricity has on cells and human tissues and explain new electrical treatment modalities. This paper draw attention the physiological changes in which the electricity generally produces biological effects and the pathological events especially in cancer, potential therapeutic effects. Most studies in literature have been done with extremely low frequency and pulsed electric fields. In terms of diseases, most studies are with glioblastoma and melanoma.

The two most important frequencies mentioned in the literature are extremely low frequency and intermediate frequency electric waves. Two different new treatment approaches have been developed by making use of these two important frequency waves. First is tumor treating fields and the other is pulsed electric fields.

Firstly, we will discuss about the biological effects of these waves in general and the studies on this subject in the literature. Secondly we will discuss about terms about this topic.

This paper draws attention to some unknown important cellular effects of electricity, and these effects are likely to create new treatment modalities. After more than a hundred articles we have scanned, we have confirmed that cancer cells are damaged and disrupted under a low-frequency electric field. We will explain these studies later in this article. In addition, we will talk about the four most important terms mentioned in the articles on this subject; Electroporation, 
electrochemotherapy, electrofusion, gene electrotansfer. This paper is a up-to-date article written on this subject, but it will be a great resource for beginners.

\section{METHODS}

We have scanned as '((Electricity) AND (Frequency)) OR (Disease)' OR (ELF) OR (EMF) OR (Mangetic) OR (Galvanotaxic) OR (Electroporation) OR (TTF) OR (PEF) from PubMed and we have selected 30 of that 300 articles, which publicated in the high quality journals.

\section{DISCUSSION}

\section{History of Electricity and First Researchs about Its Biological Effects}

An electric field is be formed by charged particles. Electric fields are discussed in physics, electrostatics and electrodynamics. Electrostatics is related to unchanged electric fields; electrodynamics is related to changes in electric fields. In addition, electrical fields have an effect on magnetic current. Electric currents can be used to generate a magnetic field, and magnetic fields can be used to generate an electric current. The electric field equation is defined as $E=F / q$, in which $E$ represents the energy of the electric field, $F$ represents the power of the particle, and $q$ represents the charge of the particle. The force of an electric field is the units named in honor of Newton and Charles-Augustin de Coulomb. The Danish physicist and chemist Hans Christian Oersted (1777-1851) was the first one who noticed the electric fields are affecting magnetism while teaching a course at the University of Copenhagen. When he passed an electric wire over a magnetic compass, he noticed that the compass needle was moving. He then made experiments that confirmed the relationship between electricity and magnetism, which showed that electric fields have a magnetic effect. Electric fields and magnetic fields were intertwined. Faraday's work was extended by the Scottish mathematician James Clerk Maxwell (1831-79), who elaborated the relationship between electricity and magnetism. Maxwell's equations, built on Faraday's law, clearly state the relationship between magnetism and electricity and show how magnetism and electricity interact individually and how they interact with each other. Maxwell's equations are the cornerstone of electromagnetic theory. (1)

Galvani proved that there is electricity in living things by showing the sciatic nerve by touching the muscle of a frog by showing its muscle twitching. However, scientists had to wait until the 1940s to prove that there was an electrical potential in their membranes, and that some cells (excitable cells such as nerve and muscle) could quickly change this potential to create an action potential. The reason for this was that the cells studied were very narrow in diameter $(20 \mu)$, but the electrodes connected to electronic devices had a much larger tip diameter. Galvani's work was confirmed by the creation of new instruments and the interest of new scientists, leading him to be regarded as the father of electrophysiology. (2)

After thorough research, a large-scale nerve cell was found in cuttlefish. Hodgkin and Huxley demonstrated the presence of membrane potential in the cell membrane for the first time by introducing electrodes into the giant nerve fiber (approximately $1000 \mu$ diameter) of this fish. According to this measure, there was a potential difference of approximately $-70 \mathrm{mV}$ between the inside and outside of a nerve cell, even at rest. (3)

The passage of matter through the cell membrane, a barrier separating the cell from the external environment, is by diffusion. The membrane potential in all cells is between 60 and $90 \mathrm{mV}$ in excitable cells such as nerve and muscle. Membrane potential found in all living cells may change temporarily in some cells and become temporary (action) potential. (4) 


\section{Intermediate Frequency and Extremely Low Frequency Electric Field's Pathopsyhological Effects}

In general, there are two different wave types in the literature. These are extremely low frequency fields and intermediate frequency fields. The effects of these two on the biologic cells are very diffrent. Using these effects, new treatment methods have been developed. Firstly, we will discuss about the biological effects of these waves in general and the studies on this subject in the literature.

For one research electric field's pathological effects on tumor cells are Significant slowdown in tumor growth in the electric field of 100-300 KHZ (intermediate frequency) and extensive destruction of tumor cells within 3-6 days. The first effect is on the mitotic cycle, the second effect is a sudden disintegration of the cell. These effects occur in 24 hours per day in 3-6 days. This research has been tested in animal malignant tumors and cell cultures. (5) For one experimental study, interestingly, in contrast to the highly metastatic rat prostate cancer cell line, a weak metastatic cell line did not respond at similar EF strengths. (6) These waves can be used in the treatment of cancer, and although it is controversial, it can be a cause of cancer. It was observed that childhood leukemia increases with in the region's of strong electric fields. There are many supporting studies similar to this. (7) Chronic 60 hertz electric field ( 30 days, 20 hours a day) When applied to reduce the production of pineal gland melatonin, it reduces to zero. As a result, estrogen production increases (melatonin suppresses estrogen production) and hence the mass or cancer in the breast grows. (8) It applies an oscillating force to each of the free ions of an oscillating external electric field, which is located on both sides of the membrane transition proteins. This external oscillation force causes a forced vibration of each free ion. When the forced vibration of the ions exceeds some critical amplitude, the pulsating ions may give a false signal to open and close the voltage-gated (even mechanically gated) channels, the disrupting the electrochemical balance of the plasma membrane and ultimately terminating the whole cell function. (9) At medium frequency, the low-density electric field inhibits mitosis cleavage by exhibiting antimicrotubular properties. $(10,11)$

ELFs have direct effects at the level of the cell membrane. They induce redistribution of membrane receptors for transferrin and low-density lipoprotein. It was concluded that each hair cell contains its own electrical resonance mechanism, which constitutes most of the frequency selectivity of the receptor potential.

The two most important frequencies mentioned in the literature are extremely low frequency and intermediate frequency electric waves. Two different new treatment approaches have been developed by making use of these two important frequency waves. First is tumor treating fields and the other is pulsed electric fields.

Some research with erythrocytes; under isotonic conditions, exposure of human erythrocytes to a high voltage pulse of several $\mathrm{kV} / \mathrm{cm}$ leads to the completion hemolysis of red cells. Experiments performed here show that hemolysis results from the effect of the electric field. (12) Vibration of the erythrocyte suspension with voltage can create a transmembrane potential across the cell membrane and, at a critical point, open the pores in the red cells. The erythrocyte pores in isotonic saline are small. They allow the passage of potassium and sodium ions, but not the sucrose and hemoglobin molecules. (13) The pores are larger under low ionic conditions and allow sucrose molecules to pass, but under no circumstances can hemoglobin pass as a direct result of the voltage pulse. Because it's a big molecule. Kinetic measurements show that hemolysis of erythrocytes follows a step-by-step mechanism: leakage of ions leads to an osmotic imbalance, leading to a colloidal hemolysis of red cells. $(14,15,16)$

Literature datas show that the ELF (extremely low electrical field) reduces the level of lipid peroxide in an oxidative stress rat. (17) 
The properties tissue electricity are likely to change in malignancies. Prostate adenocarcinoma can be treated with electrotherapy. Because the malignant cells are strong galvanotactic, can remove the diseased gland by applying small dc voltages. $(18,19,20)$

The electrical frequency of most cells in human tissues are different. For example, slow oscillations at theta frequencies $(4-12 \mathrm{~Hz}$ ) are consistently recorded in the hippocampus during working tasks, spatial navigation, and storage of episodic memory. The hippocampus is capable of generating its own theta rhythm when isolated in vitro Another treatment hypothesis in this regard is to destroy the cell by resonating the cell with electrical frequency matching. (21)

\section{Pulsed Electromangetic Field Therapy (Extremely low frequency)}

ELF- Pulsed electromagnetic field therapy (PEMFT) uses extremely low frequency electromagnetic fields in an attempt to especially glioblastoma, fractures and depression. By 2007 the FDA had approved several such stimulation devices.

Unlike TTF, pulsed electromangetic fields have been done in many more areas. There are many promising studies on depression and fracture, postoperative pain.

PEMF upregulates $X$-linked inhibitor of apoptosis, which is considered the most potent caspase inhibitor. (22) PEF (Pulsed electric fields) causes cell and organelle disruption in many cells. (23) In one PEF study ; cells were exposed to external direct current electric fields with physiological force, their reactions were recorded by light microscopy and analyzed by a quantitative monitoring method.

Voltage-gated $\mathrm{Na}+$ channel activity was pharmacologically modulated using a specific channel blocker (tetrodotoxin) or opener (veratridine) concentrations. $(24,25,26,27)$ The results showed that high metastatic MAT-LyLu cells ( $\mathrm{Na}-\mathrm{K}$ channel open or excess) reacted very strongly to the application of the electric field by migrating to the cathode. In contrast, weak metastatic At- 2 cells (with NA-K channel closed) did't respond to this. (28)

One electrical model for biological cells has allowed us to predict that the electrical field interaction with intracellular structures is increased in pulses that last longer than the charging time of the outer membrane. Experimental studies in which human cells were exposed to pulsed electric fields up to $300 \mathrm{nV} / \mathrm{cm}$ amplitude, as short as $10 \mathrm{~ns}$, confirmed this hypothesis.(PEF) The observed effects are the degradation of intracellular granule membranes without permanent damage to the cell membrane, abrupt increases in intracellular free calcium levels and increased expression of genes. In increasing electric fields, the application of submicron second envelopes evokes apoptosis (programmed cell death) in biological cells, which has been shown to reduce the growth of tumors. The possible effects of intracellular electro-effect are controlling gene function in the nucleus, controlling cell function due to calcium release (which causes cell immobilization) and regressing tumors. (29)

To summarize nanosecond pulsed electrical effects on endothelial cell's data, appropriate pulse parameters are able to enhance cell proliferation on endothelial cells which may involve the intracellular kalsiyum concertation, as well as ROS and NO production in nsPEFs treatment system. Some results suggest that nsPEFs may have a potential application in the process of accelerating the stent endothelialization. (30)

\section{Tumor Treating Fields (TTF)}

Tumor treating field is low intensity and intermediate frequency $(200 \mathrm{kHz})$ alternating electric fields, which have been demonstrated to disrupt mitosis, induce cell arrest, induce apoptosis with antimitotic properties in a variety of tumor types.

A clinical TTF device is approved in the United States and Europe for the treatment of recurrent glioblastoma. The device can be used in conjunction with regular patterns of care for patients, but are 
only available in certain treatment centers, and specific training and certification on the part of the prescribing physician.(32)

It has recently been shown in some researchs that low density, intermediate frequency and electric fields inhibit cancer cell growth by an in vitro anti-microtubule mechanism of action. By using implanted electrodes, tumor areas were also shown to inhibit the growth of dermal tumors in mice.(TTF) This study extends these findings to additional cell lines and animal tumor models using externally isolated electrodes. $(33,34,35)$ These findings have led to the initiation of a pilot clinical trial of the effects of TT fields in 10 patients with recurrent glioblastoma (GBM). In terms of disease progression, this time is normally more than twice the life expectancy. The only side effect was mild contact dermatitis. (TT fields = tumor treating fields) $(36,37,38,39,40,41)$

TTF is selectively toxic to proliferating cells via an anti-mitotic mechanism has been widely reported. (42) This may prevent damage to other cells.

\section{Membrane Electroporation Phenomenon}

It is the change in the membrane permeability of the cells in the electric field. Therapeutic models are currently being developed using this phenomenon. Electrochemotherapy is a new therapeutic option for patients with locally spread melanoma. It is based on the phenomenon of reversible electroporation. It was showed in vitro, that electroporation can prompt the formation of reactive oxygen species (ROS) near cell membranes, which can be detrimental to cell viability. $(43,44,45)$

\section{Electrochemotherapy}

Electrochemotherapy (ECT) is a locally chemotherapy that combines the administration of chemotherapeutic drugs with electric pulses for cell membrane electroporation (EP). Electroporation is a well-known phenomenon that occurs at the cell membrane when cells are exposed to high intensity electric pulses. Reversible electroporation is used to introduce drugs or genetic material into the cell without affecting cell viability. Electrochemotherapy refers to a combined treatment; electroporation and drug injection to enhance its cytotoxic effect. $(46,47)$

\section{Electrofusion}

Cell electrofusion is a safe method that can be used for preparing hybrid cells for human therapy. Electrofusion involves application of short high-voltage electric pulses to cells that are in close contact. Application of short, high-voltage electric pulses causes destabilization of cell plasma membranes. Destabilized membranes are more permeable for different molecules and also prone to fusion with any neighboring destabilized membranes. Electrofusion is a convenient method to achieve a fusion of very different cells in vitro. (48)

\section{Gene Electrotransference}

For some diseases, if gene therapy is performed using an electrical effect, it is called gene electrotransference. Another application of electroporation is the clinical trials that have been achieved, where electroporation is used for transfection of cells within tissues with plasmid DNA, for the purpose of cancer therapy. $(49,50,51)$

\section{CONCLUSIONS}

As a result, we discussed the effects of electricity on cell in general with new treatment methods. The effects of electricity on cells are very important for medicine. New treatment models were made using these effects.

As a result of literature review, we found that especially extremely low electric frequency and intermediate frequency fields have very important pathophysiological effects. We have mentioned about four important expressions on this subject; electroporation, electrofusion, electrochemotherapy, gene electrotransference. Two different new treatment approaches have 
been developed by making use of these two important frequency waves. First is tumor treating fields and the other is pulsed electric fields.

The findings in this article will provide up-to-date information on new studies on antioxidane system, atherosclerosis and especially tumor treatment. This paper will help to find some new treatment methods in the future.

In future studies, we aim to prevent the chemically damaged, uncontrolled division of tumors by electrical resonance, or otherwise possible division. After dozens of articles, we have confirmed that cancer cells are damaged and damaged under low frequency electric and pulsed electric field.

In general, we concluded that electrical fields have important physiological effects on cell biology. We hope that the topics discussed in detail in the discussion section will provide a basic source of information for future studies.

\section{ABBREVIATIONS}

EF: Electrical fields

PKC: Protein kinaz

TTF: Tumor treating fields

ELF: Extremely low frequency

EMF:Electromangetic frequency

PEF: Pulsed Electrical Fields

CONFLICT OF INTEREST

The authors confirm that this article content has no conflict of interest.

\section{ACKNOWLEDGEMENTS}

We acknowledge Ozlem Yalcinkaya for his contribution to the correction of this review article.

\section{REFERENCES}

1) Pasachoff, (2015) The history of electromagnetic theory through the lives of its founders, $N$. Metascience, 24: 233. https://doi.org/10.1007/s11016-014-9982-8

2) Kazamel M1, Warren PP2, (2017)History of electromyography and nerve conduction studies:A tribute to the founding fathers. J Clin Neurosci. 2017 Sep;43:54-60. doi: 10.1016/j.jocn.2017.05.018.

3) W. Ragsdale, A triz. Yan Wang and Clifton (2019), Cephalopod Nervous System Organization Subject: Invertebrate Neuroscience, DOI: 10.1093/acrefore/9780190264086.013.181

4) Chrysafides SM, Sharma S. (2019) Physiology, Resting Potential. In: StatPearls [Internet]. Treasure Island (FL): StatPearls Publishing; 2019 Jan-. Available from:

https://www.ncbi.nlm.nih.gov/books/NBK538338/

5) Giladi, Rosa S, Voloshin, (December 2015) Mitotic Spindle Disruption by Alternating Electric Fields Leads to Improper Chromosome Segregation and Mitotic Catastrophe in Cancer Cells https://doi.org/10.1038/srep1804610.1038/srep18046

https://www.nature.com/articles/srep18046\#supplementary-information 
6)Song, K., Im, S. H., Yoon, Y. J., Kim, H. M., Lee, H. J., \& Park, G. S. (2018). A 60 Hz uniform electromagnetic field promotes human cell proliferation by decreasing intracellular reactive oxygen species levels. PloS one, 13(7), e0199753. doi:10.1371/journal.pone.0199753

7)Crespi, C. M., Vergara, X. P., Hooper, C., Oksuzyan, S., Wu, S., Cockburn, M., \& Kheifets, L. (2016). Childhood leukaemia and distance from power lines in California: a population-based case-control study. British journal of cancer, 115(1), 122-128. doi:10.1038/bjc.2016.142

8)Fenga, C. (2016). Occupational exposure and risk of breast cancer . Biomedical Reports, 4, 282-292. https://doi.org/10.3892/br.2016.575

9) Panagopoulos D, Johansson, Carlo, (2015) Polarization: A Key Difference between Man-made and Natural Electromagnetic Fields, in regard to Biological Activity, Scientific Reports, 14914 https://doi.org/10.1038/srep14914

10)Burri, S. H., Gondi, V., Brown, P. D., \& Mehta, M. P. (2018). The Evolving Role of Tumor Treating Fields in Managing Glioblastoma: Guide for Oncologists. American journal of clinical oncology, 41(2), 191-196. doi:10.1097/COC.0000000000000395

11)Trusheim, J., Dunbar, E., Battiste, J., Iwamoto, F., Mohile, N., Damek, D., ... Connelly, J. (2017). A state-of-the-art review and guidelines for tumor treating fields treatment planning and patient follow-up in glioblastoma. CNS oncology, 6(1), 29-43. doi:10.2217/cns-2016-0032

12)Varani, K., Vincenzi, F., Ravani, A., Pasquini, S., Merighi, S., Gessi, S., ... Cadossi, R. (2017).

Adenosine Receptors as a Biological Pathway for the Anti-Inflammatory and Beneficial Effects of Low Frequency Low Energy Pulsed Electromagnetic Fields. Mediators of inflammation, 2017, 2740963. doi:10.1155/2017/2740963

13)Manser, M., Sater, M. R., Schmid, C. D., Noreen, F., Murbach, M., Kuster, N., ... Schär, P. (2017). ELF-MF exposure affects the robustness of epigenetic programming during granulopoiesis. Scientific reports, 7, 43345. doi:10.1038/srep43345

14)Vincenzi, F., Targa, M., Corciulo, C., Gessi, S., Merighi, S., Setti, S., ... Varani, K. (2013). Pulsed electromagnetic fields increased the anti-inflammatory effect of $A_{2} A$ and $A_{3}$ adenosine receptors in human T/C-28a2 chondrocytes and hFOB 1.19 osteoblasts. PloS one, 8(5), e65561. doi:10.1371/journal.pone.0065561

15) Watson, T. C., Obiang, P., Torres-Herraez, A., Watilliaux, A., Coulon, P., Rochefort, C., \& Rondi-Reig, L. (2019). Anatomical and physiological foundations of cerebello-hippocampal interaction. eLife, 8 , e41896. doi:10.7554/eLife.41896

16) Du, E; Qiang, Yuhao; Liu, Jia. (2018.) "Erythrocyte Membrane Failure by Electromechanical Stress." Appl. Sci. 8, no. 2: 174.

17)Zhang, Y., Dong, F., Liu, Z., Guo, J., Zhang, J., \& Fang, J. (2018). Nanosecond pulsed electric fields promoting the proliferation of porcine iliac endothelial cells: An in vitro study. PloS one, 13(5), e0196688. doi:10.1371/journal.pone.0196688

18) Grys, M., Madeja, Z., \& Korohoda, W. (2017). Avoiding the side effects of electric current pulse application to electroporated cells in disposable small volume cuvettes assures good cell survival. Cellular \& molecular biology letters, 22, 1. doi:10.1186/s11658-016-0030-0

19)Muratori, C., Pakhomov, A. G., Heller, L., Casciola, M., Gianulis, E., Grigoryev, Pakhomova, O. N. (2017). Electrosensitization Increases Antitumor Effectiveness of Nanosecond Pulsed Electric Fields In Vivo. Technology in cancer research \& treatment, 16(6), 1533034617712397. Advance online publication. doi:10.1177/1533034617712397 
20)Steuer, A., Wolff, C. M., von Woedtke, T., Weltmann, K. D., \& Kolb, J. F. (2018). Cell stimulation versus cell death induced by sequential treatments with pulsed electric fields and cold atmospheric pressure plasma. PloS one, 13(10), e0204916. doi:10.1371/journal.pone.0204916

21)Hummos, A., \& Nair, S. S. (2017). An integrative model of the intrinsic hippocampal theta rhythm. PloS one, 12(8), e0182648. doi:10.1371/journal.pone.0182648

22)Iwasa, K., \& Reddi, A. H. (2018). Pulsed Electromagnetic Fields and Tissue Engineering of the Joints. Tissue engineering. Part B, Reviews, 24(2), 144-154. doi:10.1089/ten.TEB.2017.0294

23)Dorsey, B. M., Cass, C. L., Cedeño, D. L., Vallejo, R., \& Jones, M. A. (2018). Effects of Specific Electric Field Stimulation on the Release and Activity of Secreted Acid Phosphatases from Leishmania tarentolae and Implications for Therapy. Pathogens (Basel, Switzerland), 7(4), 77. doi:10.3390/pathogens 7040077

24)Beebe S.J. (2015) Considering effects of nanosecond pulsed electric fields on proteins Bioelectrochemistry, 103 , pp. 52-59.

25)Rosazza, C., Meglic, S. H., Zumbusch, A., Rols, M. P., \& Miklavcic, D. (2016). Gene Electrotransfer: A Mechanistic Perspective. Current gene therapy, 16(2), 98-129.

doi:10.2174/1566523216666160331130040

26)Morotomi-Yano K., Akiyama H., Yano K.-I. (2014) Different involvement of extracellular calcium in two modes of cell death induced by nanosecond pulsed electric fields, Archives of Biochemistry and Biophysics, 555-556, pp. 47-54.

27)Hanna, H., Andre, F. M., \& Mir, L. M. (2017). Electrical control of calcium oscillations in mesenchymal stem cells using microsecond pulsed electric fields. Stem cell research \& therapy, 8(1), 91. doi:10.1186/s13287-017-0536-z

28)Borys P. (2012). On the biophysics of cathodal galvanotaxis in rat prostate cancer cells: Poisson-Nernst-Planck equation approach. European biophysics journal : EBJ, 41(6), 527-534. doi:10.1007/s00249-012-0807-7

29)Steuer, A., Wolff, C. M., von Woedtke, T., Weltmann, K. D., \& Kolb, J. F. (2018). Cell stimulation versus cell death induced by sequential treatments with pulsed electric fields and cold atmospheric pressure plasma. PloS one, 13(10), e0204916. doi:10.1371/journal.pone.0204916

30)Hanna, H., Denzi, A., Liberti, M., André, F. M., \& Mir, L. M. (2017). Electropermeabilization of Inner and Outer Cell Membranes with Microsecond Pulsed Electric Fields: Quantitative Study with Calcium Ions. Scientific reports, 7(1), 13079. doi:10.1038/s41598-017-12960-w

31)Zhu, P., \& Zhu, J. (2017). Tumor treating fields: a novel and effective therapy for glioblastoma: mechanism, efficacy, safety and future perspectives. Chinese Clinical Oncology, 6(4).

doi:10.21037/cco.v6i4.16215

32)Mrugala MM, Engelhard HH, Dinh Tran D, et al. (2014) Clinical practice experience with NovoTTF-100A ${ }^{\mathrm{TM}}$ system for glioblastoma: The Patient Registry Dataset (PRiDe). Semin Oncol ; 41 Suppl 6:S4.

33)Hottinger, A. F., Pacheco, P., \& Stupp, R. (2016). Tumor treating fields: a novel treatment modality and its use in brain tumors. Neuro-oncology, 18(10), 1338-1349. doi:10.1093/neuonc/now182

34)Carr, L., Bardet, S. M., Burke, R. C., Arnaud-Cormos, D., Leveque, P., \& O'Connor, R. P. (2017).

Calcium-independent disruption of microtubule dynamics by nanosecond pulsed electric fields in U87 human glioblastoma cells. Scientific reports, 7, 41267. doi:10.1038/srep41267 
35)Berzingi, S., Newman, M., \& Yu, H. G. (2016). Altering bioelectricity on inhibition of human breast cancer cells. Cancer cell international, 16, 72. doi:10.1186/s12935-016-0348-8

36) Pakhomova, O. N., Gregory, B. W., Semenov, I., \& Pakhomov, A. G. (2013). Two modes of cell death caused by exposure to nanosecond pulsed electric field. PloS one, 8(7), e70278. doi:10.1371/journal.pone.0070278

37) Yung-Shin, (2017), Studying Electrotaxis in Microfluidic Devices, Sensors, 17(9), 2048; https://doi.org/10.3390/s17092048,

38)Huang, Y. J., Hoffmann, G., Wheeler, B., Schiapparelli, P., Quinones-Hinojosa, A., \& Searson, P. (2016). Cellular microenvironment modulates the galvanotaxis of brain tumor initiating cells. Scientific reports, 6, 21583. doi:10.1038/srep21583

39)Huang, Y. J., Schiapparelli, P., Kozielski, K., Green, J., Lavell, E., Guerrero-Cazares, H., ... Searson, P. (2017). Electrophoresis of cell membrane heparan sulfate regulates galvanotaxis in glial cells. Journal of cell science, 130(15), 2459-2467. doi:10.1242/jcs.203752

40) Staresinic, B., Jesenko, T., Kamensek, U., Krog Frandsen, S., Sersa, G., Gehl, J., \& Cemazar, M. (2018). Effect of calcium electroporation on tumour vasculature. Scientific reports, 8(1), 9412. doi:10.1038/s41598-018-27728-z

41)Kıvrak, E. G., Yurt, K. K., Kaplan, A. A., Alkan, I., \& Altun, G. (2017). Effects of electromagnetic fields exposure on the antioxidant defense system. Journal of microscopy and ultrastructure, 5(4), 167-176. doi:10.1016/j.jmau.2017.07.003

42)Kim, E. H., Song, H. S., Yoo, S. H., \& Yoon, M. (2016). Tumor treating fields inhibit glioblastoma cell migration, invasion and angiogenesis. Oncotarget, 7(40), 65125-65136.

doi:10.18632/oncotarget.11372

43) Wichtowski, M., \& Murawa, D. (2018). Electrochemotherapy in the treatment of melanoma. Contemporary Oncology/Współczesna Onkologia, 22(1), 8-13.

https://doi.org/10.5114/wo.2018.74387

44)Bian, S., Zhou, Y., Hu, Y., Cheng, J., Chen, X., Xu, Y., \& Liu, P. (2017). High-throughput in situ cell electroporation microsystem for parallel delivery of single guide RNAs into mammalian cells. Scientific reports, 7, 42512. doi:10.1038/srep42512

45)Shi, J., Ma, Y., Zhu, J., Chen, Y., Sun, Y., Yao, Y., ... Xie, J. (2018). A Review on Electroporation-Based Intracellular Delivery. Molecules (Basel, Switzerland), 23(11), 3044. doi:10.3390/molecules23113044

46)Probst, U., Fuhrmann, I., Beyer, L., \& Wiggermann, P. (2018). Electrochemotherapy as a New Modality in Interventional Oncology: A Review. Technology in cancer research \& treatment, 17, 1533033818785329. doi:10.1177/1533033818785329

47)Sersa, G., Teissie, J., Cemazar, M., Signori, E., Kamensek, U., Marshall, G., \& Miklavcic, D. (2015). Electrochemotherapy of tumors as in situ vaccination boosted by immunogene electrotransfer. Cancer immunology, immunotherapy : CII, 64(10), 1315-1327. doi:10.1007/s00262-015-1724-2

48) Trontelj, K., Usaj, M., \& Miklavcic, D. (2010). Cell electrofusion visualized with fluorescence microscopy. Journal of visualized experiments : JoVE, (41), 1991. doi:10.3791/1991

49) Rosazza, C., Meglic, S. H., Zumbusch, A., Rols, M. P., \& Miklavcic, D. (2016). Gene Electrotransfer: A Mechanistic Perspective. Current gene therapy, 16(2), 98-129.

doi:10.2174/1566523216666160331130040 
50)Znidar, K., Bosnjak, M., Semenova, N., Pakhomova, O., Heller, L., \& Cemazar, M. (2018). Tumor cell death after electrotransfer of plasmid DNA is associated with cytosolic DNA sensor upregulation.

Oncotarget, 9(27), 18665-18681. doi:10.18632/oncotarget.24816

51)Lesueur, L. L., Mir, L. M., \& André, F. M. (2016). Overcoming the Specific Toxicity of Large Plasmids Electrotransfer in Primary Cells In Vitro. Molecular therapy. Nucleic acids, 5(3), e291.

doi:10.1038/mtna.2016.4 